\title{
Large-Scale Noise-Resilient Evolution-Strategies
}

\author{
Oswin Krause \\ University of Copenhagen, Department of Computer Science \\ Copenhagen, Denmark \\ oswin.krause@di.ku.dk
}

\begin{abstract}
Ranking-based Evolution Strategies (ES) are efficient algorithms for problems where gradient-information is not available or when the gradient is not informative. This makes ES interesting for Reinforcement-Learning (RL). However, in RL the high dimensionality of the search-space, as well as the noise of the simulations make direct adaptation of ES challenging. Noise makes ranking points difficult and a large budget of re-evaluations is needed to maintain a bounded error rate. In this work, the ranked weighting is replaced by a linear weighting function, which results in nearly unbiased stochastic gradient descent (SGD) on the manifold of probability distributions. The approach is theoretically analysed and the algorithm is adapted based on the results of the analysis. It is shown that in the limit of infinite dimensions, the algorithm becomes invariant to smooth monotonous transformations of the objective function. Further, drawing on the theory of SGD, an adaptation of the learning-rates based on the noise-level is proposed at the cost of a second evaluation for every sampled point. It is shown empirically that the proposed method improves on simple ES using Cumulative Step-size Adaptation and ranking. Further, it is shown that the proposed algorithm is more noise-resilient than a ranking-based approach.
\end{abstract}

\section{CCS CONCEPTS}

- Theory of computation $\rightarrow$ Evolutionary algorithms; Continuous optimization; Random search heuristics; • Computing methodologies $\rightarrow$ Reinforcement learning;

\section{KEYWORDS}

CMA-ES, Evolution Strategies, Reinforcement-Learning, Large-Scale, Stochastic Optimization

\section{ACM Reference Format:}

Oswin Krause. 2019. Large-Scale Noise-Resilient Evolution-Strategies. In Genetic and Evolutionary Computation Conference (GECCO '19), fuly 13-17, 2019, Prague, Czech Republic. ACM, New York, NY, USA, 9 pages. https: //doi.org/10.1145/3321707.3321724

Permission to make digital or hard copies of all or part of this work for personal or classroom use is granted without fee provided that copies are not made or distributed for profit or commercial advantage and that copies bear this notice and the full citation on the first page. Copyrights for components of this work owned by others than the author(s) must be honored. Abstracting with credit is permitted. To copy otherwise, or republish, to post on servers or to redistribute to lists, requires prior specific permission and/or a fee. Request permissions from permissions@acm.org.

GECCO '19, July 13-17, 2019, Prague, Czech Republic

(C) 2019 Copyright held by the owner/author(s). Publication rights licensed to the Association for Computing Machinery.

ACM ISBN 978-1-4503-6111-8/19/07 ..\$15.00

https://doi.org/10.1145/3321707.3321724

\section{INTRODUCTION}

This paper is concerned with finding the solution of the problem

$$
\arg \min _{x} f(x), x \in \mathbb{R}^{d},
$$

where $f: \mathbb{R}^{d} \rightarrow \mathbb{R}$ is a black-box function with minimum obtained at $x^{*} \in \mathbb{R}^{d}$. For this type of problem, ranking-based Evolution Strategies (ES) proved to be among the most successful algorithms with the CMA-ES [4] and its variants forming the state-of-the-art. Ranking-based algorithms do not use the function values directly, but instead rank the candidate points by function-value and only use the ranking information to improve on current solutions. This allows the algorithm to be invariant to monotonic transformations of the objective function, including non-differentiable and even noncontinuous transformations, which are difficult to handle otherwise.

It is difficult to make ES noise-resilient. The lower bound of the convergence-rate in terms of total number of function evaluations $T$ has been shown to be $\Omega(1 / \sqrt{T})$ [2]. The lower bound relies on the property that ESs adapt the step-size to be $O\left(\left\|x-x^{*}\right\| / d\right)$. It has been shown that ensuring a larger step-size allows for $O(1 / T)$ convergence on a quadratic function, the theoretical optimum $[2,8]$.

For rank-based algorithms, noisy observations make the optimization problem difficult, as the noise will introduce errors in the ranking. Without additional noise-handling, the algorithm will approach a random walk as soon as the noise dominates the observed difference in function values. There have been a number of approaches to solve this. Most notable are approaches that use re-evaluation of points, especially racing-based algorithms [6] and algorithms based on the observed difference of ranks under reevaluation [3]. For these approaches, it can be observed that the number of re-evaluations increases so quickly that additional iterations are too expensive to carry out. This holds especially for racing-based algorithms as they necessarily rely on loose frequentist bounds.

Another recent approach [7] is to estimate the slope of the progress in average function value and to use a hypothesis test to check whether the number of re-evaluations need to be increased. It has been shown that the full algorithm has $O(1 / T)$ convergence rate $[7,8]$. The noise-handling approach of sampling additional points instead of re-evaluation has the advantage that it is compatible with other measures of function difficulty as presented in [14], where the number of sampled points is automatically increased on a function which is considered more difficult, e.g. multi-modal. However, the approach of [7] has the flaw that instead of weighted ranking, only selection of the better half of the points is performed. This ignores most of the information in the function values and leads to slow linear convergence rate in regions where the noise is small compared to the observed difference in function values. Additionally, using the noise-ratio of the measured slope does not 
generalize well to ill-conditioned und multi-modal functions, where the observed slope can be naturally much smaller than the observed variation in function values.

In this paper, we explore a novel direction with focus towards medium to large-scale applications with thousands of variables, for example direct policy-search in reinforcement-learning [5]. We focus our attention on ES employing isotropic Gaussian distributions and function values corrupted by additive noise. Instead of ranking, we will use the original function values and devise a linear weighting function which normalizes the observed function values locally. This linearity allows for the observed errors to be averaged out. Additionally, we will draw from the field of Stochastic Gradient Descent algorithms (SGD), mostly used in large-scale machine-learning to devise an adaptation of the learning rates instead of increasing the number of sampled points. It is known from standard SGD literature [17] that when $f$ is smooth and strongly convex and if the additive noise has bounded variance, a learningrate schedule of $O(1 / T)$ leads to convergence rate $O(1 / T)$. For ES, this result can only hold if the necessary requirement of a large enough step-size is met, as a shrinking step-size can be equivalently interpreted as growing variance of the noise, violating the condition of bounded variance in SGD. Even if this condition is met, estimating the optimal schedule is hard and while convergence is guaranteed, the wrong schedule will lead to suboptimal performance. In this work, we define the schedule implicitely using an estimate of the signal-to-noise ratio of the observed variance in function values. This requires each point to be evaluated twice. In contrast to [7], our approach of measuring noise does not rely on additional assumptions on the shape of the function.

This paper is structured as follows: The necessary background on ES is introduced in Section 2. Our novel algorithm is described in Section 3. Section 4 describes the experiments and the results are reported and discussed in Section 5. Finally we conclude the paper in Section 6.

\section{EVOLUTION-STRATEGIES}

Evolution-Strategies minimize $f$ using a search-distribution, which is often chosen as a normal distribution $p\left(x \mid \mu_{t}, \sigma_{t}^{2} C_{t}\right)$. Throughout this paper, we assume the search-distribution to be isotropic, $C_{t}=I_{d}$. In the following, we will use the Information-GeometricFlow (IGO) theory to describe the behaviour of the ES [15]. The achievement of this theory is the realization that large parts of the update dynamics of an ES can be described as following the natural gradient on a locally reweighted objective function. Following the IGO-flow, an iteration $t$ of the algorithm can be described as optimizing the objective function

$$
\max _{\mu_{t}, \sigma_{t}} E_{p\left(x \mid \mu_{t}, \sigma_{t}^{2} I_{d}\right)}\left\{w_{t}(f(x))\right\},
$$

where $w_{t}: \mathbb{R} \rightarrow \mathbb{R}$ is a weighting function chosen by the algorithm at every step. In each iteration, a sample $x_{1, t}, \ldots, x_{\lambda, t}$ is drawn from the distribution via

$$
x_{i, t}=\mu_{t}+\sigma_{t} z_{i, t}, z_{i, t} \sim \mathcal{N}\left(0, I_{d}\right) .
$$

The sampled points are evaluated on the weighted objective function to obtain $w_{i, t}=w_{t}\left(f\left(x_{i, t}\right)\right)$. The weight function is often chosen as a weighted quantile function

$$
w(y)=\omega(P(y<f(x))),
$$

which assigns larger weights to points when the probability of sampling a better point is small. Typically $\omega$ is chosen to perform selection of the better half of points, which amounts to assigning a weight of zero to the worse half of points. In practice, a biased estimator is used which ranks the points by function value and assigns a weight $w_{i, t}$ based on this rank.

The standard update of $\mu_{t}$ is derived by computing a step of natural gradient descent wrt. $\mu_{t}$ in (1):

$$
\mu_{t+1}=\mu_{t}+\alpha_{t} \sum_{i}^{\lambda} w_{i, t}\left(x_{i, t}-\mu_{t}\right)
$$

With $\sum_{i} w_{i, t}=1, w_{i, t} \geq 0$ and learning-rate $\alpha_{t}=1$ this simplifies to the well known weighted mean of samples

$$
\mu_{t+1}=\sum_{i}^{\lambda} w_{i, t} x_{i, t} \text {. }
$$

The IGO-Flow theory predicts that $\sigma_{t}$ is also updated using natural gradient descent, in the same manner as the update of $\mu_{t}$. However, in practice this assigns too small values to $\sigma_{t}$ as the value of (1) has a tendency to decrease when $\sigma_{t}$ decreases, while the length of the update of $\mu_{t}$, as given by (2), scales with $\sigma_{t}$. Instead, heuristics are used to update the step-size.

The Cumulative Step-size Adaptation algorithm (CSA) [16] is the current state-of-the-art used in many variants of the CMA-ES algorithm. In direct comparisons, it performed better than almost all alternative heuristics [11]. The CSA is based on measuring the autocorrelation of the steps $\mu_{t+1}-\mu_{t}$ over a certain time-horizon. When steps are detected to be correlated, the step-size will be increased to allow for larger steps while steps are decreased when steps are anti-correlated. In this work, we use the most recent formulation [1], which has an in-built mechanism to handle varying learning-rates.

The CSA accumulates an evolution path, a long-term average of previous steps, and compares its length with the length expected for an unbiased random walk. The update of the evolution path $p_{t}$ is given by

$$
p_{t+1}=\left(1-c_{p, t}\right) p_{t}+\sqrt{c_{\sigma}\left(1-c_{p, t}\right) \mu_{\mathrm{eff}, t}} \frac{\mu_{t+1}-\mu_{t}}{\sigma_{t}} .
$$

Here, $\mu_{\text {eff, } t}$ is the effective sample-size and $c_{p, t}$ is a learning-rate. The update rule is chosen such that when $\frac{\mu_{t+1}-\mu_{t}}{\sigma_{t}} \sim \mathcal{N}\left(0, I_{d}\right)$, $p_{t+1} \sim \mathcal{N}\left(0, I_{d}\right)$. This update depends on the exact phrasing of the update-rule used in (2), as a change of it would also change the distribution of steps. To make the update of the evolution path independent from the mean-update, it can be rephrased in terms of $z_{i, t}$, which assumes that steps taken are similar to (3)

$$
p_{t+1}=\left(1-c_{p, t}\right) p_{t}+\sqrt{c_{p, t}\left(1-c_{p, t}\right) \mu_{\mathrm{eff}, t}} \sum_{i=1}^{\lambda} w_{i} z_{i, t} .
$$

Our goal is to compare the length of the path with its expectation under standard-normal distributed steps. However, the expected length changes under varying learning-rate, and is naturally smaller in the first few iterations when the path is initialized with $p_{0}=0$. 
To handle this, another variable is introduced which tracks the expected squared length:

$$
\gamma_{\sigma, t+1}=\left(1-c_{p, t}\right)^{2} \gamma_{\sigma, t}+c_{p, t}\left(1-c_{p, t}\right) .
$$

Finally, we can compare the length of the path with the expected length, and obtain as an update of the step size

$$
\sigma_{t+1}^{2}=\sigma_{t}^{2} \exp \left(\frac{c_{\sigma, t}}{d_{\sigma, t}}\left(\frac{\left\|p_{t+1}\right\|}{\chi(d)}-\sqrt{\gamma_{\sigma, t+1}}\right)\right) .
$$

\section{A NEW, NOISE-RESILIENT ES}

In this section, we will reformulate the simple ES introduced in the previous section based on linear weights. We will first introduce the linear weights and than investigate the core components of the algorithms for necessary changes. To do this, we will analyse parts of the algorithm on simple linear or quadratic functions. In the end, we will bring the different parts together to form a single algorithm. Core aspects of the analysis will be the update of the mean and adaptation of learning rates on a noisy function.

\subsection{Linear Weight-Functions}

If the weight function is linear, i.e. $w_{t}(y)=-a_{t}\left(y-b_{t}\right)$, for $a_{t}, b_{t} \in$ $\mathbb{R}$, it is easier to make the resulting algorithm noise-resilient, as independent noise on the function-values can be averaged out and the averages follow an approximate normal distribution. With this choice, the objective (1) can be rewritten as

$$
E_{p\left(x \mid \mu_{t}, \sigma_{t}\right)}\left\{w_{t}(f(x))\right\}=w_{t}\left(E_{p\left(x \mid \mu_{t}, \sigma_{t}\right)}\{f(x)\}\right) .
$$

Thus, a (natural) gradient step with this weight function is just a rescaled version of the original (natural) gradient. The bias term $b_{t}$ vanishes in the expectation of the gradient, however it guides the variance of the estimate as we see below.

By using linear weights the ES loses invariance to monotonic transformations. Still, a local linear normalization is surprisingly strong in higher dimensions. To see this, assume $f$ has the form $f(x)=g\left(x^{T} x\right), x \in \mathbb{R}^{d}$, where $g$ is a smooth, monotonic increasing function. Further assume $\left\|\mu_{t}\right\|=1$. It is known that in high dimensions the optimal step size of a rank-based ES on sphere in dimension $d$ is (e.g. [9, Lemma 10])

$$
\sigma_{t}=O\left(\frac{\left\|\mu_{t}\right\|}{d}\right)=O\left(\frac{1}{d}\right) .
$$

Therefore, the expected squared distance of the samples from the mean is

$$
E\left\{\left\|x-\mu_{t}\right\|^{2}\right\}=\sigma_{t}^{2} d=O\left(\frac{1}{d}\right) .
$$

Thus, when $d \rightarrow \infty, E\left\{\left\|x-\mu_{t}\right\|^{2}\right\} \rightarrow 0$. This allows approximation of $g$ around $\mu_{t}^{T} \mu_{t}$ via a first-order Taylor expansion, provided that the dimensionality is large enough to drive the step-size sufficiently small. Linear weights allow normalization of this expansion to a fixed slope and offset, inducing local invariance to the first-order taylor-expansion. Similar results can be expected to hold for more general classes of functions, as long as the notion of an ascent direction as generalization of the gradient makes sense.

With linear weights, we can not apply sample-selection anymore, however, we can shift the weight towards successful samples using an apropriate value of $b_{t}$. On a simple quadratic function, $f(x)=$
$x^{T} x$, the optimal $b_{t}$ can be computed easily. When fixing $a_{t}=1$, the estimator of the natural gradient of $\mu_{t}$ becomes

$$
-E_{p(x)}\left\{\left(f(x)-b_{t}\right)\left(x-\mu_{t}\right)\right\} .
$$

Simple algebraic transformations reveal that the second moment of this estimator is minimized at

$$
\arg \min _{b_{t}} E_{p(x)}\left\{\left(f(x)-b_{t}\right)^{2}\left\|x-\mu_{t}\right\|^{2}\right\}=(d+2) \sigma_{t}^{2}+\left\|\mu_{t}\right\|^{2} .
$$

This value is only slightly larger than the average function value $d \sigma_{t}^{2}+\left\|\mu_{t}\right\|^{2}$. Thus, allowing weights to be negative can reduce the variance of the estimator. However, it is difficult to find the proper normalization factor for the CSA path-update (4) for negative weights. We argue that a weight function should therefore have the properties that the

- Weights should not be negative

- Best samples should have the largest impact on the estimate

- Sum of absolute values should be close to one

This can be estimated from past iterations, but a biased estimate can be achieved using

$$
w_{t}(y)=\frac{y-\max _{i} f\left(x_{i, t}\right)}{\sum_{j=1}^{\lambda}\left[f\left(x_{j, t}\right)-\max _{i} f\left(x_{i, t}\right)\right]} .
$$

This gives the worst sample a weight of zero, the best sample the largest weight and all weights are guaranteed to sum to one.

\subsection{Update of the Mean}

We argue that the update equation of the mean in equation (2) is not optimal for linear weights that sum to one. Instead, we propose that the subtracted mean in the second term should be replaced by the sample mean

$$
\mu_{t+1}=\mu_{t}+\alpha_{t} \sum_{i}^{\lambda} w_{i, t}\left(x_{i, t}-\frac{1}{\lambda} \sum_{j=1}^{\lambda} x_{j, t}\right) .
$$

This update does not introduce additional bias, but has a smaller estimation error than the original update rule. To see this, let $x_{w}, t$ indicate the point in the sample with $f\left(x_{w, t}\right)=\max _{t} f\left(x_{i, t}\right)$. If we assume a very small step-size $\sigma_{t}, f$ can be approximated around $\mu_{t}$ by a linear function $f_{t}(x)=g_{t}^{T}\left(x-\mu_{t}\right)+c$. If we apply equation (8) to this approximation, we obtain the sample-weights:

$$
\begin{aligned}
w_{k, t} & \approx \frac{g_{t}^{T}\left(x_{w, t}-\mu_{t}\right)-g_{t}^{T}\left(x_{k, t}-\mu_{t}\right)}{\sum_{j=1}^{\lambda}\left(x_{w, t}-\mu_{t}\right)-\left(x_{j, t}-\mu_{t}\right)} \\
& =\frac{g_{t}^{T}\left(z_{w, t}-z_{k, t}\right)}{\lambda \cdot g_{t}^{T}\left(z_{w, t}-\frac{1}{\lambda} \sum_{j=1}^{\lambda} z_{j, t}\right)}
\end{aligned}
$$

The denominator, $d_{t}$, can be interpreted as an estimate of the slope of the function of size $O(\lambda|g|)$. With this, (2) can be rewritten as

$$
\mu_{t+1}=\mu_{t}+\alpha_{t} \frac{\sigma_{t}}{d_{t}} \sum_{i}^{\lambda} g_{t}^{T}\left(z_{w, t}-z_{k, t}\right) z_{i, t} .
$$

Due to finite sample sizes, $\tilde{z}_{t}=\frac{1}{\lambda} \sum_{j=1}^{\lambda} z_{j, t}$ is small, but not zero. While this error in the sampling of the first order statistics will 
cancel in the difference $z_{w, t}-z_{k, t}$ and will therefore not affect the weights, it will impact the estimation of the gradient

$$
\begin{gathered}
\mu_{t+1}=\mu_{t}+\alpha_{t} \frac{\sigma_{t}}{d_{t}} \sum_{i}^{\lambda} g_{t}^{T}\left(z_{w, t}-z_{k, t}\right)\left(z_{i, t}-\tilde{z}_{t}\right) \\
+\alpha_{t} \frac{\sigma_{t}}{d_{t}} \sum_{i}^{\lambda} g_{t}^{T}\left(z_{w, t}-z_{k, t}\right) \tilde{z}_{t} .
\end{gathered}
$$

As weights sum to one, we can simplify the last term and obtain

$$
\mu_{t+1}=\mu_{t}+\alpha_{t} \sigma_{t} \tilde{z}_{t}+\alpha_{t} \frac{\sigma_{t}}{d_{t}} \sum_{i}^{\lambda} g_{t}^{T}\left(z_{w, t}-z_{k, t}\right)\left(z_{i, t}-\tilde{z}_{t}\right) .
$$

By applying the correction in (9), we remove the $\alpha_{t} \sigma_{t} \tilde{z}_{t}$ term, which reduces the variance of the gradient step, without affecting the expectation. As the removed term does not depend on the sampled function values, but only on the sampled points, this decreases the variance in the gradient direction.

This correction does not work with the original non-linear rankbased weights, as the ranks depend on the sampled median ${ }^{1}$. For weights that sum to zero, the old and new update are equivalent. Further, the derivation presented here only holds for very small step-sizes. In reality, $\sigma_{t}$ is always tuned such that at least the secondorder term in the expansion can not be ignored. Our results can be extended to this case by noting that $\left\|\tilde{z}_{t}\right\|$ is on average a factor $O(1 / \sqrt{\lambda})$ smaller than the length of the $z_{i, t}$ and thus its contribution to the error function is small compared to the other terms.

\subsection{Noise-Handling}

In this section, we want to incorporate active mechanisms to handle additive noise. Instead of function values $f\left(x_{i, t}\right)$, we observe corrupted values $f\left(x_{i, t}\right)+\epsilon_{i, t}$, where $\epsilon_{i, t}$ is an unobserved mean-free variable with variance $\operatorname{Var}\{\epsilon\}$ bounded by $V \in \mathbb{R}^{+}$.

The fact that equations (4) and (9) use averages of the linear weights already makes the algorithm resilient to noise. however, due to the small sample size, the additive noise will dominate near the optimum and the averages become progressively unrealiable This will become worse in higher dimensions. To see this, assume $f(x)=x^{T} x+\epsilon$. The Taylor expansion around $\mu_{t}$ is exact and we obtain

$$
f\left(\mu_{t}+\sigma_{t} z\right)=\sigma_{t}^{2} z^{T} z+2 \sigma_{t} \mu_{t}^{T} z+\epsilon+\text { const } .
$$

From $z \sim \mathcal{N}\left(0, I_{d}\right)$, we obtain that $z^{T} z=O(d)$ and $\left|\mu_{t}^{T} z\right|=O\left(\left\|\mu_{t}\right\|\right)$ If we further set $\sigma_{t}=O\left(\frac{\left\|\mu_{t}\right\|}{d}\right)$ we see that the whole equation is $O\left(\frac{\left\|\mu_{t}\right\|^{2}}{d}\right)+\epsilon$. Thus, for a constant variance of $\epsilon$, increasing $d$ will increase the relative impact of the noise. Moreover, the signal vanishes with $\left\|\mu_{t}\right\|^{2}$, which is caused by a simultaneous decrease of the slope of the function and a decrease of the step length, both in $O\left(\left\|\mu_{t}\right\|\right)$. Thus, the signal-to-noise ratio will shrink as $O\left(\frac{\left\|\mu_{t}\right\|^{2}}{d V}\right)$ which only allows for $O\left(\frac{1}{\sqrt{T}}\right)$ convergence rate in number of function evaluations $T$.

\footnotetext{
${ }^{1}$ We tested this correction for rank-based weights and found a degradation in performance.
}

If we use the linear weight function (8), we can treat (9) as stochastic gradient descent (SGD) on the manifold of probability distributions. Thus, we can guarantee convergence to the optimum by reducing the learning rate $\alpha_{t}$ over time. We propose to adapt $\alpha_{t}$ by estimating how much of the variance observed in the sampled function-values can be explained by noise. To formalize this, let us define $\operatorname{Var}\{f(X)\}$ as the variance of the uncorrupted function values, i.e. the variance observed simply by sampling different $X$ and our goal is to find an estimator for $\operatorname{Var}\{f(X)\} / \operatorname{Var}\{\epsilon\}$. For this, we evaluate each point twice and obtain samples $f^{(1)}\left(x_{i, t}\right)$ and $f^{(2)}\left(x_{i, t}\right)$. From the samples, we define the two random variables $U$ and $V$ with values

$$
u_{i, t}=\frac{f^{(1)}\left(x_{i, t}\right)+f^{(2)}\left(x_{i, t}\right)}{2}, v_{i, t}=\frac{f^{(1)}\left(x_{i, t}\right)-f^{(2)}\left(x_{i, t}\right)}{2} .
$$

It is easy to see that $\operatorname{Var}\{f(X)\}+\operatorname{Var}\{\epsilon\}=\operatorname{Var}\{U\}+\operatorname{Var}\{V\}$ and that $U$ and $V$ are independent. Moreover, $V$ is mean-free and $\operatorname{Var}\{V\}=\operatorname{Var}\{\epsilon\} / 2$ and thus

$$
\frac{\operatorname{Var}\{f(X)\}}{\operatorname{Var}\{\epsilon\}}=\frac{\operatorname{Var}\{U\}}{2 \operatorname{Var}\{V\}}-\frac{1}{2} .
$$

Again, due to the small sample size, we will use time-averaged estimates of the empirical variances with empirical mean $\hat{u}_{t}$ :

$$
\begin{aligned}
& \operatorname{Var}_{V, t+1}=\left(1-c_{\epsilon, t}\right) \operatorname{Var}_{V, t}+c_{\epsilon, t} \frac{1}{\lambda} \sum_{i=1}^{\lambda} v_{i, t}^{2} \\
& \operatorname{Var}_{U, t+1}=\left(1-c_{\epsilon, t}\right) \operatorname{Var}_{U, t}+c_{\epsilon, t} \frac{1}{\lambda-1} \sum_{i=1}^{\lambda}\left(u_{i, t}-\hat{u}_{t}\right)^{2}
\end{aligned}
$$

And finally, the global learning-rate is chosen as

$$
\alpha_{t}=1-\frac{1}{\frac{\operatorname{Var}_{U, t+1}}{2 \operatorname{Var}_{V, t+1}}-\frac{1}{2}}
$$

To make use of the lower variances of the averages, we compute the weights as $w_{i, t}=w_{t}\left(u_{i, t}\right)$. Accordingly, we compute $w_{t}$ as

$$
w_{t}(u)=\frac{u-\max _{i} u_{i, t}}{\sum_{j=1}^{\lambda}\left[u_{j, t}-\max _{i} u_{i, t}\right]} .
$$

\subsection{Complete Algorithm}

In this section, we combine the different parts discussed in the previous sections and unify the noisy and noise-less cases to form the fCSA algorithm, presented in Algorithm 1. The tuned learningrates are given in Table 1. As tuning of ES is a complicated task, the learning-rates presented here are not intended to be optimal and often ad-hoc choices. We have tuned the algorithm only in the range $1000 \leq d \leq 16000$ with the default value of $\lambda$. but we are confident that it still works for higher dimensions and modest increases of $\lambda$.

To reduce the cost of noise-handling in regimes of the parameter space where the noise does not significantly impact the algorithm performance, we flip a coin with a probability $r_{t}$ depending on the detected noise-level, which we choose to reduce the additional function-evaluations to $10 \%$ in those regions. We correct for this by adapting the learning-rate $c_{\epsilon, t}$ with importance-sampling. We use $\alpha_{t}$ as a global learning rate, allowing for longer accumulation times or smaller steps. We scale $c_{\epsilon, t}$ with $\alpha_{t-1}^{3 / 2}$ because with lower signalto-noise ratio longer accumulation times are needed to obtain a 


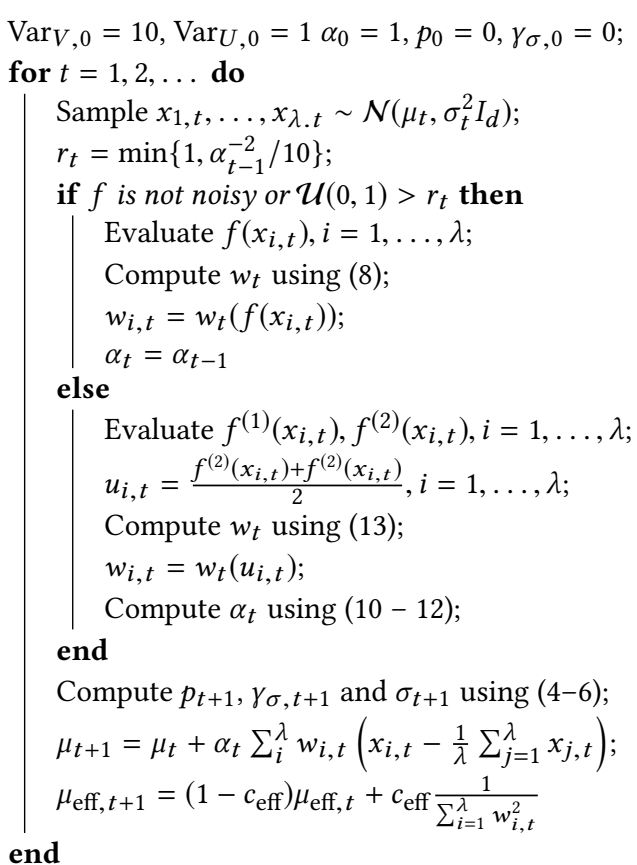

Algorithm 1: The fCSA Algorithm. Hyper-parameters are given in Table 1.

Table 1: Learning-rates used by the fCSA. Note that some parameters need to be computed before the evaluation of points.

$$
\begin{aligned}
\text { Variable } & \text { Value at step } t \\
\hline \lambda & =4+3\lfloor\log (d)\rfloor \\
c_{\epsilon, t} & =\frac{\alpha_{t-1}^{3 / 2}}{100 r_{t}} \\
c_{\text {eff }} & =\frac{1}{100} \\
c_{p, t} & =2 \frac{\mu_{\text {eff }, t}+2}{d+\mu_{\text {eff }, t}+5} \\
d_{\sigma, t} & =\frac{\sqrt{c_{p, t}\left(1-c_{p, t}\right)}}{2 \alpha_{t} c_{p, t}}
\end{aligned}
$$

reliable estimate and there is a direct interaction between $\alpha_{t}$ and quality of estimate which requires a more conservative tuning of $c_{\epsilon, t}$.

We use the original CSA in this algorithm, except a different tuning of $d_{\sigma, t}$, which cancels the muliplied correction factor in (4) As a correlation between noise and estimated sample-size $\mu_{\mathrm{eff}, t}$ can lead to a bias of the expected step-length in the CSA, we compute the estimated sample-size as a time-average over several iterations, using the estimate of the first iteration as initial guess for $\mu_{\text {eff, } 0}$.

\section{EXPERIMENTS}

We compared the fCSA to a set of baseline-algorithms. First, we implement a simple ranking-based ES as outlined in Section 2. We will refer to this as CSA. Next, we implemented the pcCMSA algorithm [7, 8] using a reference implementation provided by the authors ${ }^{2}$. Our implementation differs in two ways from $[7,8]$ :

\footnotetext{
${ }^{2}$ Available at https://gi thub.com/hellwigm/pcCMSA-ES
}

Table 2: Learning-rates used by the CSA. All parameters are independent of $t$. For $w_{i, t}$, we assume the indices of the points to be ordered by the ranks of function-values.

\begin{aligned} Variable & Value at step $t \\$\hline CSA & \\ \hline$w_{i, t} & =\frac{\max \{0, \log (\lambda / 2+1 / 2)-\log (i)\}}{\sum_{j=1}^{\lambda} \max \{0, \log (\lambda / 2+1 / 2)-\log (j)\}} \\ \mu_{\mathrm{eff}, t} & =\frac{1}{\sum_{i=1}^{\lambda} w_{i, t}^{2}} \\ c_{p, t} & =2 \alpha_{t} \frac{\mu_{\mathrm{eff}, t}+2}{d+\mu_{\mathrm{eff}, t}+5} \\ d_{\sigma, t} & =\frac{1}{4}\left(1+c_{p, t}\right) \\$\hline $\mathrm{pcSA} & \\$\hline$L & =3 d \\ \bar{\alpha} & =0.05 \\ v & =1 / 3 \\ \mu_{0} & =2+\lfloor\log (d)\rfloor \\ c & =2 \\ b & =1.5 \\ \tau_{\sigma} & =(2 d)^{-1 / 2}\end{aligned}$

Table 3: List of functions used in the experiments. In all experiments, $\kappa=10^{-2}$ and $\epsilon \sim \mathcal{N}\left(0,10^{-4}\right)$.

\begin{tabular}{lll} 
Function & $f(x)$ & Init \\
\hline Sphere & $\sum_{i=1}^{d} x_{i}^{2}$ & $\mathcal{N}\left(0, I_{d}\right)$ \\
NoisySphere & $\sum_{i=1}^{d} x_{i}^{2}+\epsilon$ & $\mathcal{N}\left(0, I_{d}\right)$ \\
NoisyEllipsoid & $\sum_{i=1}^{d} \kappa^{-\frac{i-1}{d-1}} x_{i}^{2}+\epsilon$ & $\mathcal{N}\left(0, I_{d}\right)$ \\
Cigar & $\sum_{i=2}^{d} x_{i}^{2}+\kappa x_{1}^{2}$ & $\mathcal{N}\left(0, I_{d}\right)$ \\
Discus & $\kappa \sum_{i=2}^{d} x_{i=x_{1}^{2}}^{2}$ & $\mathcal{N}\left(0, I_{d}\right)$ \\
Ellipsoid & $\sum_{i=1}^{d} \kappa^{-\frac{i-1}{d-1}} x_{i}^{2}$ & $\mathcal{N}\left(0, I_{d}\right)$ \\
LogSphere & $\log \left(\sum_{i=1}^{d} x_{i}^{2}\right)$ & $\mathcal{N}\left(0, I_{d}\right)$ \\
LogDiscus & $\log \left(\kappa \sum_{i=2}^{d} x_{i}^{2}+x_{1}^{2}\right)$ & $\mathcal{N}\left(0, I_{d}\right)$
\end{tabular}

a) we set the covariance matrix to the identity matrix in every iteration and $b$ ) we use an extension provided by the reference implementation, which replaces the test for a linear trend by the non-parametric Mann-Kendall test for trend ${ }^{3}$. The latter renders the algorithm invariant under monotonous transformations of the objective function while not changing the performance compared to the results reported in $[7,8]$. We used the parameters as provided by the reference implementation, with the exception of $L=3 d$ which lead to better results than the $L=5 d$ used in the reference.

As another baseline, we implement the pcfCSA, a re-evaluation based version of the fCSA which uses the Mann-Kendal test to adapt the number of evaluations similar to the pcCMSA. To gauge the overall effect of noise-handling in the fCSA, we implement the fCSA-NNH (No Noise Handling), where $\alpha_{t}=1$ and only a single evaluation per point is performed. Finally, as ablation study, we implemented a version of the fCSA using the standard mean-update in equation (3), which we refer to as fCSAM.

All hyper-parameters are given in Tables $1 \& 2$. The pcfCSA uses the combination of hyper-parameters of the pcSA and fCSA

\footnotetext{
${ }^{3}$ This change was suggested in personal communication with the authors
} 


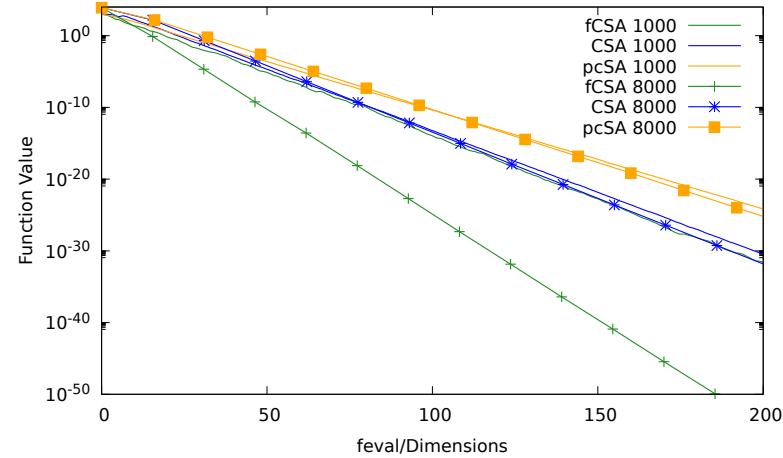

(a) Sphere

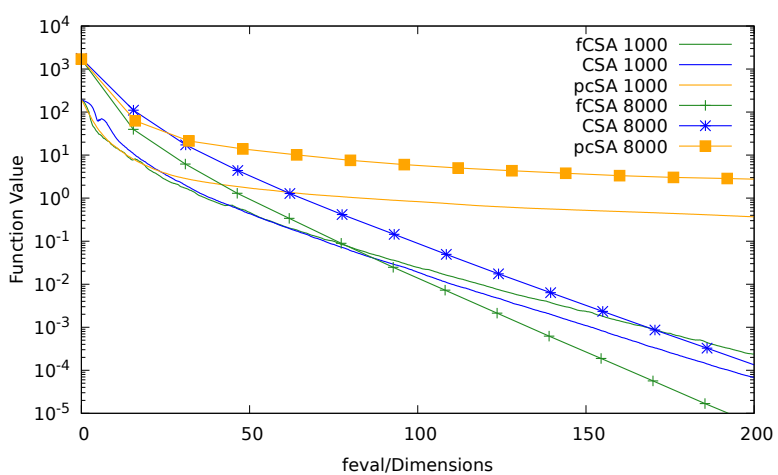

(b) Ellipsoid

Figure 1: Representative runs of the fCSA, pcSA and CSA algorithms on Sphere and Ellipsoid with $d=1000,8000$. Shown are $f\left(\mu_{t}\right)$ over the number of function evaluations divided by $d$. Note that the curve of fCSA on Sphere with $d=1000$ is nearly hidden behind the curves of CSA.

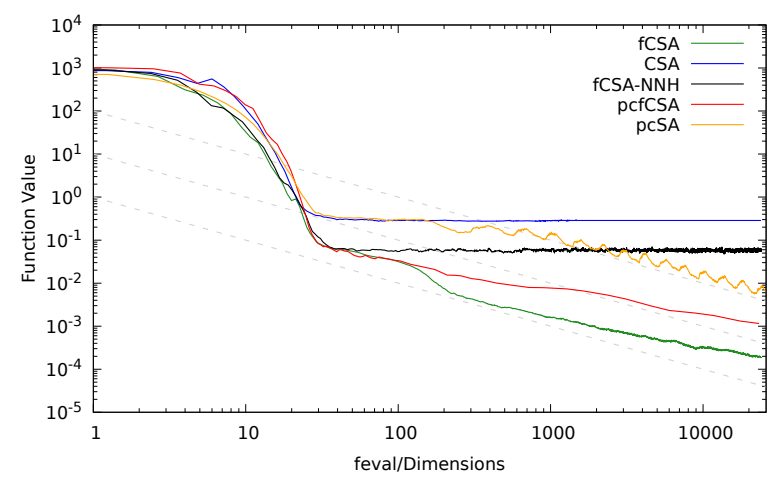

(a) Sphere, $d=1000$

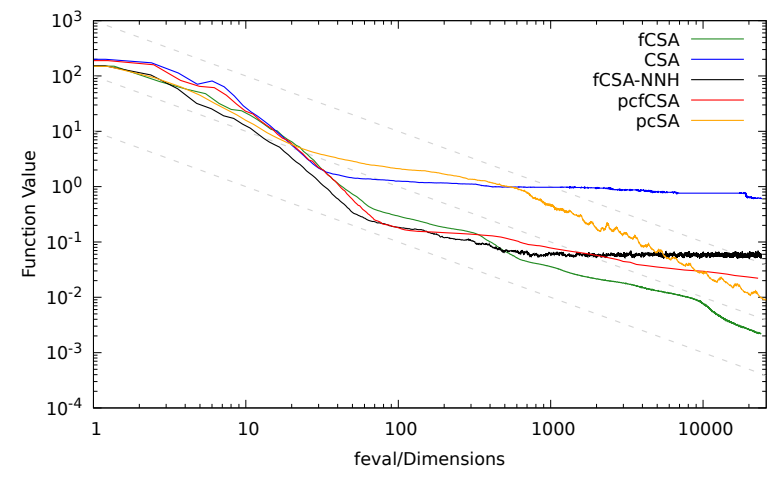

(c) Ellipsoid, $d=1000$

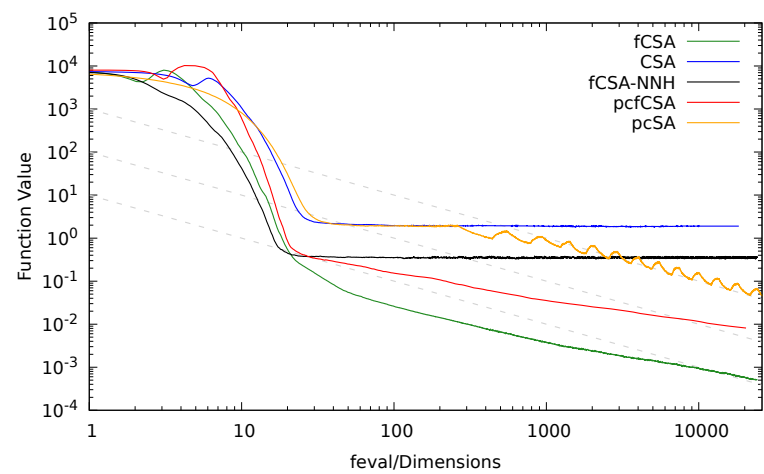

(b) Sphere, $d=8000$

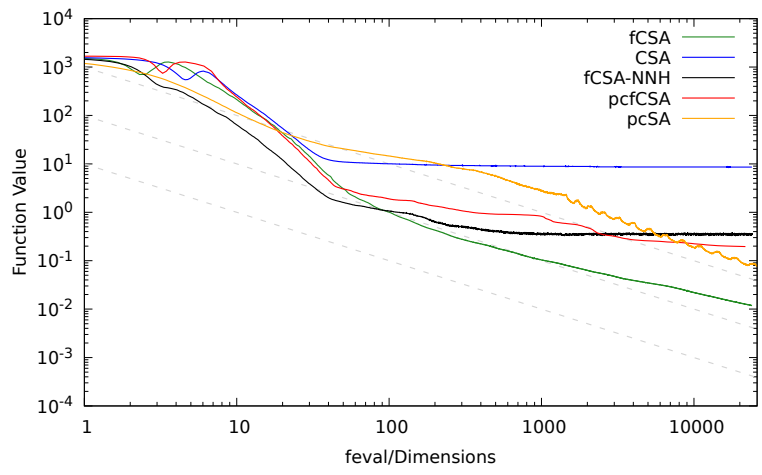

(d) Ellipsoid, $d=8000$

Figure 2: Typical runs of the proposed algorithm and the base-lines on NoisySphere and NoisyEllipsoid with $d=1000,8000$. Shown are results of the fCSA without noise-handling (fCSA-NNH), with adapted learning rates (fCSA) and using re-sampling (pcfCSA), as well as the CSA and pcSA. Plotted are $\left\|\mu_{t}\right\|^{2}$ over the number of function evaluations divided by $d$. Gray-dotted lines indicate $O(1 / T)$ trend-lines

algorithms, with the exception of $L=100$. In all experiments we chose as initial step-size $\sigma_{0}=3 / \sqrt{d}$, which mostly skips the initial adaptation phase of $\sigma_{t}$. We did not compare the behaviour at significantly smaller values of $\sigma_{0}$, because initial experiments have shown no difference in behaviour of the algorithms. All algorithms except pcSA start with $\lambda=4+\lfloor 3 \log (d)\rfloor$. For the pcSA, we set $\mu_{0}=\lceil\nu \lambda\rceil$. 
As test-functions, we considered the functions in Table 3. Our main goal was to compare the algorithms on Sphere in the noise-less and noisy cases. Those results tell us how the algorithm behaves when the function is approximately spherical. As this is a strong assumption, we additionally investigated a set of mildly conditioned quadratic functions, presented in Table 3.

To analyse the noise-less case, we ran the fCSA, fCSAM, pcSA and CSA on the quadratic noise-less functions in Table 3 with dimensions $d=1000 \cdot 2^{i}, i=0, \ldots, 4$. For this experiment, we deactivate the noise-handling procedures for all approaches. We measured the number of function-evaluation needed to reach a target precision $10^{-7}$ and averaged the results over 10 trials, each starting with a different starting-point.

To investigate the impact of monotonic transformations, we compared the fCSA on Sphere and Discus with their log-transformed counter-parts. We conducted the experiments under the same conditions as before, except the stopping-condition. As the logarithm is not bounded from below, we exchanged the stopping condition to a function-value smaller than $\log \left(10^{-7}\right)$.

Finally, we compared all algorithms except fCMAM on the NoisySphere and NoisyEllipsoid functions with noise-variance $10^{-4}$. In this experiment we only compare $d=1000$ and $d=8000$ and show single, representative runs with a fixed budget of function evaluations of $1000 \lambda d$.

\section{RESULTS AND DISCUSSION}

The results of the noise-less experiments are shown in Figure 3 and single representative runs on Sphere and Ellipsoid are shown in Figure 1. On Sphere (Figure 3a), using the sample-mean instead of the true mean (fCSAM) reduced the number of function evaluations by a factor of two in low dimensions, and a factor of four in higher dimensions. Most notably, the pcSA, CSA and fCSAM require function evaluations that increase linearly with $d$. While the fCSA also requried comparably more evaluations, it did not increase at the same rate as the CSA and required $40 \%$ less function evaluations than the CSA at $d=16000$. This scaling of the fCSA could be an effect of sub-optimal learning rates at lower dimensions.

On Cigar, Figure 3b, the fCSA was slightly faster than the CSA and significantly more than the fCSAM and pcSA algorithms. The picture is a bit more complex on Discus (Figure 3c), and Ellipsoid (Figure 3d). Here the fCSA required factor 2-3 more functionevaluations than the CSA at lower dimensions, but required less at higher dimensions. In contrast, the pcSA is nearly unaffected on Discus, but has severly decreased performance on Ellipsoid, similar to Cigar. When comparing fCSA and fCSAM, we see that in comparsion the fCSAM was less affected on Discus and Ellipsoid, which hints towards that using the sample-mean as normalization is not optimal. This makes sense, as differences in the sample-mean lead to a different noise-pattern on a non-isotropic quadratic function. Still, the fCSA performed better than the fCSAM in all our experiments.

The results of the log-transformed functions compared to the original functions are given in Figure 4. There is a small difference at $d=1000$, however it vanishes completely towards $d=16000$, as predicted. The effect is slightly stronger on Discus.
The results of the noisy experiments are shown in Figure 2. It can be seen that the CSA performed poorly in all settings and stopped making progress early in the optimization process. The fCSA-NNH reached overall lower function values compared to the CSA, but it also stagnated after some time. Adding noise-handling increased the number of function-evaluations slightly in the early regime due to the occasional use of additional function-evaluations for the fCSA.It can be seen that the re-evaluation based pcfCSA achieves a convergence-rate of $O(1 / \sqrt{T})$, while the pcSA shows the trend of $O(1 / T)$ convergence rate, but can use this advantage only far later during optimization. The fCSA shows a more complex curve with overall larger progress compared to the fCSA, but pcSA would at some point outperform it.

The saw-tooth pattern of the pcSA curves is the result of alternating between incrementing and decrementing the population size every $L$ iterations. In tuning experiments, larger values of $L$ would lead to a reduction of the pattern, while overall making less progress. Significant reduction of $L$ would lead to stagnation. Even though the pcfCSA did only use $L=100$, it did not show any of those problems. The results on Ellipsoid are similar in nature, with overall worse progress of the algorithms, as expected.

\section{CONCLUSION}

In this work, we presented a novel approach of using linear weight functions in Evolution-Strategies instead of ranking, targeted towards noisy functions in high dimensions. We showed empirically and analytically that we still enjoy invariance towards smooth monotonic transformations in the limit of infinite dimensions. Further, we showed that this approach does not perform significantly worse and sometimes even better than using weighted ranking, however it is slightly more sensitive towards ill-conditioning of the function. We think that our approach is valid in high dimensions and should be combined with other approaches to adapt the covariancematrix in medium [10] to large-scale applications [12,13].

We further introduced a novel estimator of the noise of the function and showed that adapting the learning-rate based on the noise-level can be used instead of increasing the number of samples or re-evaluations in an iteration. This realization is not new, but a result from the progress in stochastic gradient descent algorithms. While our approach leads to good results, it does not achieve the optimal convergence rate of $O(1 / T)$ unlike the pcCMSA [8]. Still, empirically it performed best given the limited budget of our experiments.

We were certainly not the first proposing to use the functionvalues directly. Most notable is the work by Uchida et al. [18], who analyzed the convergence properties of an ES using an identity weight function, i.e. using the function-values directly. Their algorithm followed the natural gradient which resulted in poor theoretical as well as experimental performance of the algorithm. Another work [19] introduced a baseline, similar to our $b_{t}$, again following the natural gradient. Our approach differs in two major ways: first, we use a linear weight function for normalization of the function value and second we use the CSA for step-size adaptation instead of the natural gradient. 


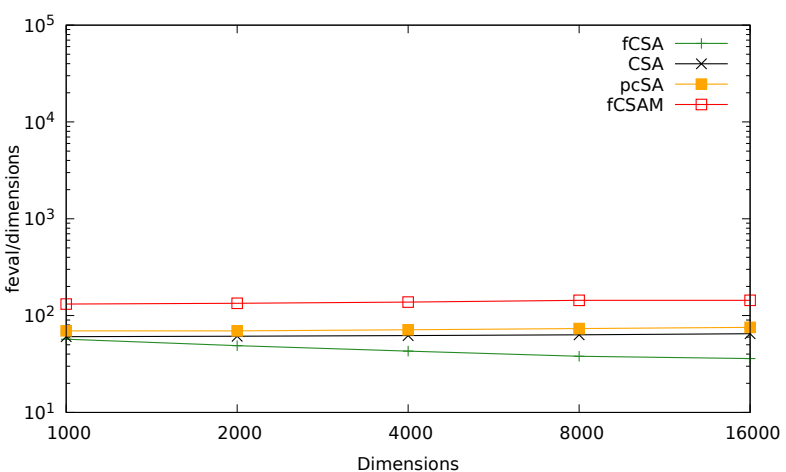

(a) Sphere

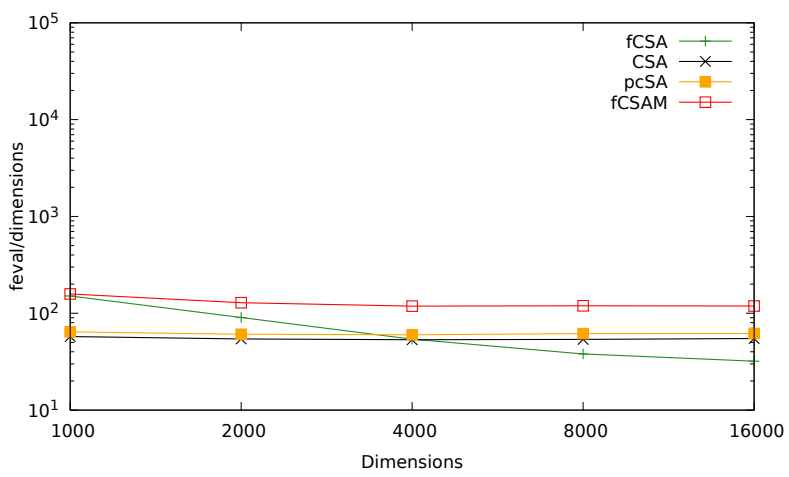

(c) Discus

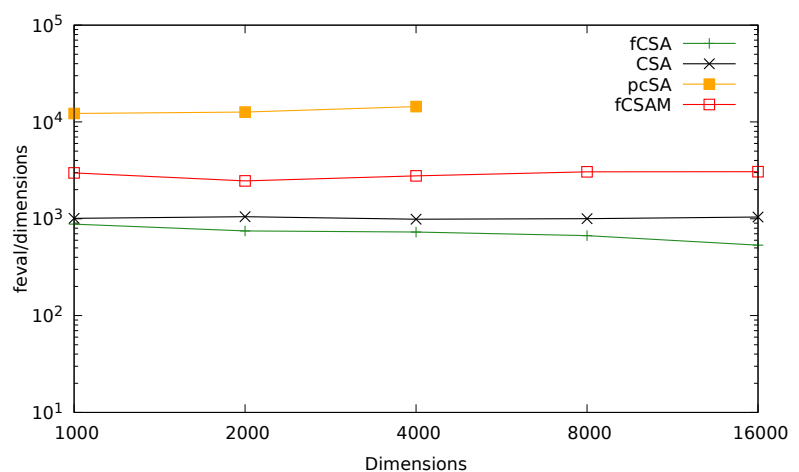

(b) Cigar

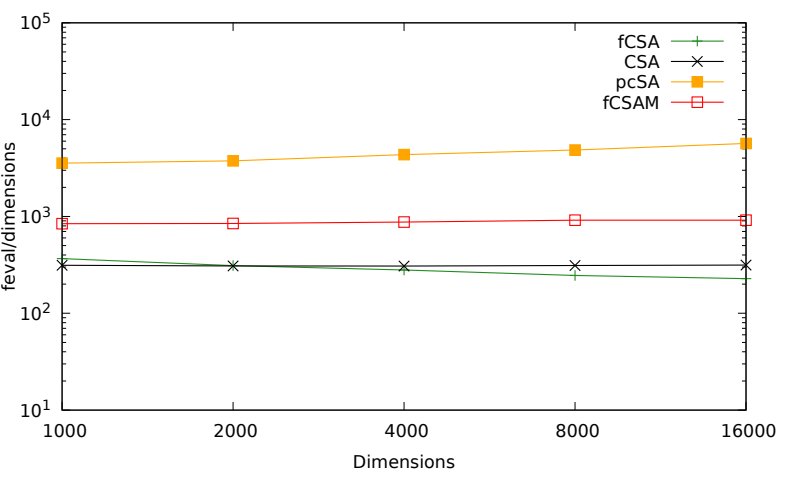

(d) Ellipsoid

Figure 3: Runtime results between CSA, fCSA, pcSA and fCSAM on quadratic benchmark functions. Shown are number of function evaluations divided by $d$ to reach function value $10^{-7}$ for $d=1000 \cdot 2^{i}, i=0, \ldots, 4$. Results are averages over 10 runs, variances are small and not shown. Missing values on Cigar did not finish in time. See Table 3 for a description of the functions.

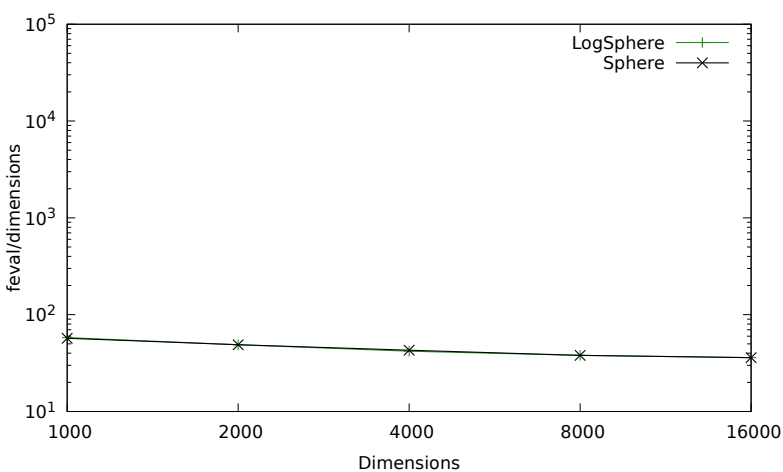

(a) LogSphere

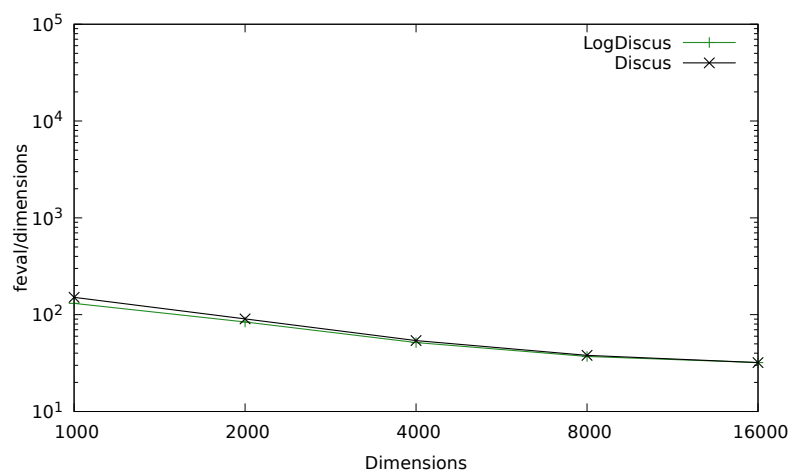

(b) LogDiscus

Figure 4: Comparison of fCSA on Sphere and Discus with their log-transformed counterparts. Shown are number of function evaluations divided by $d$ to reach function value $\log \left(10^{-7}\right)$ over $d$ for $d=1000 \cdot 2^{i}, i=0, \ldots, 4$. Results are averages over 10 runs, variances are small and not shown. See Table 3 for a description of the functions.

\section{ACKNOWLEDGMENTS}

I thank Tobias Glasmachers for the enlightening discussions, Helle Sørensen for her statistical advice and an anonymous reviewer for the extensive feedback.

\section{REFERENCES}

[1] Youhei Akimoto and Nikolaus Hansen. 2019. Diagonal Acceleration for Covariance Matrix Adaptation Evolution Strategies. (Jan. 2019). https://hal.inria.fr/ hal-01995373 working paper or preprint. 
[2] S. Astete-Morales, M. L. Cauwet, and O. Teytaud. 2015. Evolution strategies with additive noise: A convergence rate lower bound. In Proceedings of the 2015 ACM Conference on Foundations of Genetic Algorithms XIII. ACM, 76-84.

[3] N. Hansen, S. P. N. Niederberger, L. Guzzella, and P. Koumoutsakos. 2009. A Method for Handling Uncertainty in Evolutionary Optimization with an Application to Feedback Control of Combustion. IEEE Transactions on Evolutionary Computation 13, 1 (2009), 180-197.

[4] N. Hansen and A. Ostermeier. 2001. Completely derandomized self-adaptation in evolution strategies. Evolutionary Computation 9, 2 (2001), 159-195.

[5] V. Heidrich-Meisner and C. Igel. 2008. Evolution strategies for direct policy search. In International Conference on Parallel Problem Solving from Nature. Springer, 428-437.

[6] V. Heidrich-Meisner and C. Igel. 2009. Hoeffding and Bernstein races for selecting policies in evolutionary direct policy search. In Proceedings of the 26th Annual International Conference on Machine Learning. ACM, 401-408.

[7] M. Hellwig and H. G. Beyer. 2016. Evolution under strong noise: A self-adaptive evolution strategy can reach the lower performance bound-the PCCMSA-ES. In International Conference on Parallel Problem Solving from Nature. Springer, 26-36.

[8] M. Hellwig and H.-G. Beyer. 2018. On the steady state analysis of covariance matrix self-adaptation evolution strategies on the noisy ellipsoid model. Theoretical Computer Science (2018). https://doi.org/10.1016/j.tcs.2018.05.016.

[9] J. Jägersküpper. 2007. Algorithmic analysis of a basic evolutionary algorithm for continuous optimization. Theoretical Computer Science 379, 3 (2007), 329-347.

[10] O. Krause, D. R. Arbonès, and C. Igel. 2016. CMA-ES with optimal covariance update and storage complexity. In Advances in Neural Information Processing Systems. 370-378.

[11] O. Krause, T. Glasmachers, and C. Igel. 2017. Qualitative and Quantitative Assessment of Step Size Adaptation Rules. In Proceedings of the 14th ACM/SIGEVO
Conference on Foundations of Genetic Algorithms. ACM, 139-148.

[12] I. Loshchilov. 2014. A computationally efficient limited memory CMA-ES for large scale optimization. In Proceedings of the 2014 Annual Conference on Genetic and Evolutionary Computation. ACM, 397-404.

[13] I. Loshchilov, T. Glasmachers, and H. G. Beyer. 2017. Limited-Memory Matrix Adaptation for Large Scale Black-box Optimization. arXiv:arXiv:1705.06693

[14] K. Nishida and Y. Akimoto. 2016. Population size adaptation for the CMA-ES based on the estimation accuracy of the natural gradient. In Proceedings of the Genetic and Evolutionary Computation Conference 2016. ACM, 237-244.

[15] Y. Ollivier, L. Arnold, A. Auger, and N. Hansen. 2017. Information-geometric optimization algorithms: A unifying picture via invariance principles. Fournal of Machine Learning Research 18, 18 (2017), 1-65.

[16] A. Ostermeier, A. Gawelczyk, and N. Hansen. 1994. Step-size adaptation based on non-local use of selection information. In Parallel Problem Solving from Nature (PPSN). Springer, 189-198.

[17] A. Rakhlin, O. Shamir, K. Sridharan, et al. 2012. Making Gradient Descent Optimal for Strongly Convex Stochastic Optimization.. In ICML, Vol. 12. Citeseer, 15711578.

[18] K. Uchida, S. Shirakawa, and Y. Akimoto. 2018. Analysis of Information Geometric Optimization with Isotropic Gaussian Distribution Under Finite Samples. In Proceedings of the Genetic and Evolutionary Computation Conference (GECCO '18). ACM, New York, NY, USA, 897-904. https://doi.org/10.1145/3205455.3205487

[19] S. Yi, D. Wierstra, T. Schaul, and J. Schmidhuber. 2009. Stochastic search using the natural gradient. In Proceedings of the 26th Annual International Conference on Machine Learning. ACM, 1161-1168. 